\title{
Impact of electron beam quality on nonlinear harmonic generation in high-gain free-electron lasers
}

\author{
Sandra G. Biedron,* Zhirong Huang, Kwang-Je Kim, and Stephen V. Milton \\ Advanced Photon Source, Argonne National Laboratory, Argonne, Illinois 60439 \\ Giuseppe Dattoli and Alberto Renieri \\ ENEA, Divisione Fisica Applicata, Centro Ricerche Frascati, C.P. 65, 00044 Frascati, Rome, Italy \\ William M. Fawley \\ Lawrence Berkeley National Laboratory, Berkeley, California 94720 \\ Henry P. Freund \\ Science Applications International Corporation, McLean, Virginia 22102 \\ Heinz-Dieter Nuhn \\ Stanford Linear Accelerator Center, Stanford University, Menlo Park, California 94309-0210
}

Pier Luigi Ottaviani

ENEA, Divisione Fisica Applicata, Centro Ricerche E. Clementel, Via Don Fiammelli 2, Bologna, Italy

(Received 20 November 2001; published 8 March 2002)

\begin{abstract}
Nonlinear harmonic generation can be a very useful and important phenomenon for single-pass freeelectron lasers (FELs) operating in the high-gain regime. Strong bunching at the fundamental wavelength and its associated higher harmonic content allow significant radiation at shorter wavelengths to be emitted without serious effects upon the output power at the fundamental. Here, we analyze the relative sensitivities to beam quality variations of the output fundamental and harmonic powers for a visiblewavelength FEL operating in the high-gain regime.
\end{abstract}

DOI: 10.1103/PhysRevSTAB.5.030701

\section{MOTIVATION}

There is a growing consensus that the next-generation, high-brightness light sources will be based upon singlepass, high-gain, free-electron lasers (FELs) [1]. One method to achieve ultrashort wavelength $(\lambda \sim$ a few $\AA$ ) FELs for which traditional mirror technologies fail is to use self-amplified spontaneous emission (SASE) [2]. SASE originates from the random longitudinal bunching on an electron beam that is traversing a dipole undulator magnet array. The central emission radiation wavelength is related to the electron beam energy and the strength of the undulator device. Among the various methods to reach shorter wavelengths at somewhat lower electron beam energies are those that exploit effects that drive coherent bunching at higher harmonics. These include nonlinear harmonic bunching in single-undulator FELs operating in the exponential gain regime [3] starting from either SASE or a weak input signal; the high-gain harmonic generation (HGHG) scheme [4], that starts from a much stronger input signal and relies upon klystron-like energy modulation and subsequent bunching; and the two-undulator

*Corresponding author. Also at MAX-Laboratory, University of Lund, Lund, Sweden, SE-22100.

$1098-4402 / 02 / 5(3) / 030701(8) \$ 20.00$
PACS numbers: 41.60.Cr, 42.65.Ky, 52.59.-f, 42.72.Bj

harmonic generation scheme [5], which starts from SASE or a weak "master oscillator" input signal and then changes resonant wavelength to that corresponding to a higher harmonic well before the nominal saturation point. These approaches are similar in that they all require high-brightness, moderately high-energy electron beams, and high field quality undulators.

In this paper, we are concerned with nonlinear harmonic generation in the exponential gain regime of single-pass, high-gain FELs because it appears to be a promising way to produce significant radiation power at shorter wavelengths with minimal changes to the required electron beam parameters or undulator configuration. In this process, exponentially growing bunching at the fundamental wavelength leads to collateral, exponentially growing bunching at higher harmonics (both odd and even) and, eventually, nearly simultaneous saturation of both the fundamental and higher harmonic output radiation powers. For radiation emission, the odd harmonics are favored as they couple most effectively to the natural undulating motion of the electron beam through a linearly polarized undulator. Nonlinear harmonic bunching and radiation could itself be the desired final output, or, alternatively, serve as a seed for further FEL amplification at shorter wavelengths [6]. Optimization of nonlinear harmonic generation may influence the design of future light sources

(C) 2002 The American Physical Society

030701-1 
operating in the vacuum ultraviolet to x-ray regions of the spectrum and is therefore of great interest.

Our group has used three different and quite distinct numerical simulation codes to investigate the impact of electron beam quality (i.e., emittance, energy spread, and peak current) upon the predicted nonlinear harmonic output, choosing base parameters corresponding to the first phase of the Low-Energy Undulator Test Line (LEUTL) SASE FEL at the Advanced Photon Source (APS) at Argonne National Laboratory (ANL) [7]. Since nonlinear harmonic radiation generation is driven by the higher harmonic content of the strongly growing bunching at the fundamental wavelength, we expect that the sensitivity of the final harmonic output to beam quality, when compared to that of the fundamental, should not be extraordinarily great. This assertion is in contrast to earlier thoughts [8] that extrapolated upon earlier experience concerning harmonic output in the low-gain regime [9].

\section{CODE DESCRIPTIONS}

\section{A. GINGER}

GINGER [10] is a multidimensional [3D macroparticle, 2D $(r-z)$ radiation field], polychromatic FEL simulation code. The equations of motion are averaged over an undulator period following the standard Kroll-Morton-Rosenbluth [11] formulation while an eikonal approximation in time and space is used for field propagation. For nonwaveguide simulations, GINGER uses an expanding radial grid whose spacing is approximately constant near the origin but grows exponentially near the outer boundary. For polychromatic SASE simulations, GINGER can be initiated with either electron beam shot noise or, alternatively, photon noise. In monochromatic mode (which is true for nearly all the results presented here), the radiation is presumed to be at a single discrete wavelength. Macroparticle bunching in the longitudinal plane can be diagnosed through approximately the ninth harmonic (with accuracy dependent upon macroparticle statistics); however, GINGER presently calculates and propagates radiation only within a bandpass centered upon the fundamental wavelength. Thus, the effects of any emitted harmonic radiation upon the electron beam are ignored; that, in general, is a good approximation up to saturation.

\section{B. MEDUSA}

MEDUSA [12] is a 3D, multifrequency, macroparticle simulation code that represents the electromagnetic field as a superposition of Gauss-Hermite modes and uses a source-dependent expansion to determine the evolution of the optical mode radius. The field equations are integrated simultaneously with the 3D Lorentz force equations. MEDUSA differs from the other nonlinear simulation codes in that no undulator-period average is imposed on the electron dynamics. It is capable of treating quadrupole and corrector fields, magnet errors, and multiple segment undulators of various quantities and types. MEDUSA is able to treat the fundamental and all harmonics simultaneously. Because of this feature, MEDUSA is also capable of treating the full SASE problem at the fundamental and harmonics.

\section{PRometeo}

PROMETEO [13] is a 1D, multiparticle code based on a modified version of the Prosnitz, Szoke, and Neil formulation of FEL dynamical equations [14]. The original model has been generalized to include the effect of beam emittance and the undulator errors. The code is capable of accounting for the evolution of the fundamental harmonics and for the coherent generation of higher-order harmonics in SASE or oscillator FELs, including the optical klystron and segmented undulators.

\section{RESULTS}

The case study for the nonlinear harmonic generation sensitivity is the first phase of the LEUTL SASE FEL with the nominal parameters given in Table I. We began by finding the optimal operating fundamental wavelength by making scans versus wavelength to find the minimum exponential gain length. For simplicity, we adopted a single-segment undulator with curved pole-face focusing, even though the actual LEUTL configuration has 2.4-m undulator sections separated by $0.36-\mathrm{m}$ drift spaces used for external focusing, beam offset adjustment using corrector magnets, and electron and optical beam diagnostics. We also injected an input power at the fundamental of $1 \mathrm{~W}$ and used an electron beam with a Gaussian profile in transverse phase space. Table II lists the optimum wavelength for maximal gain as determined by each of the three

TABLE I. LEUTL SASE FEL parameters.

\begin{tabular}{lc}
\hline \hline & Parameters \\
\hline Wavelength (nm) & 517 \\
Photon beam radiation classification & Visible \\
Electron beam $\gamma$ & 430.53 \\
Normalized emittance (mm mrad) & $5 \pi$ \\
Energy spread (\%) & 0.1 \\
Current (A) & 150 \\
Undulator period (mm) & 33 \\
Magnetic field (T) & 1.0 \\
Undulator gap (mm) & 9.3 \\
Undulator parameter K & 3.1 \\
\hline \hline
\end{tabular}

TABLE II. Optimum fundamental gain length and wavelength.

\begin{tabular}{lcc}
\hline \hline \multicolumn{1}{c}{ Code } & Min. $L_{\text {gain }}(\mathrm{m})$ & Optimum $\lambda(\mathrm{nm})$ \\
\hline GINGER & 0.67 & 519.0 \\
MEDUSA & 0.63 & 518.8 \\
PROMETEO & 0.77 & 518.1 \\
\hline \hline
\end{tabular}


TABLE III. Fundamental saturated power.

\begin{tabular}{lc}
\hline \hline \multicolumn{1}{c}{ Code } & Power (MW) \\
\hline GINGER & 103 \\
MEDUSA & 109 \\
PROMETEO & 109 \\
\hline \hline
\end{tabular}

codes, while Table III gives the predicted radiation power at saturation. The effective FEL parameter $\rho \sim 0.0021$, which is useful for comparison with the energy spread and normalized emittance scans.

Next, we compared the output of the higher harmonics. As stated previously, GINGER calculates the output power at only the fundamental but can diagnose the electron beam bunching for higher harmonics, while MEDUSA and PROMETEO can calculate both the fundamental and the higher harmonic powers. To lowest order, the power ratios should scale as the square of the bunching ratio. For this analysis, we first determined the $z$-locations of saturation of the fundamental bunching or output power; in general, the peak in bunching occurs $\sim \frac{1}{2}-1$ gain length before the saturated power position. Then, values of the harmonic bunching or output power were calculated at this same location. Note that the harmonics do not necessarily saturate at the same point where the fundamental saturates due to the differences in the variations of phase-space rotations of the sub-microbunching. As a result, the powers quoted for the

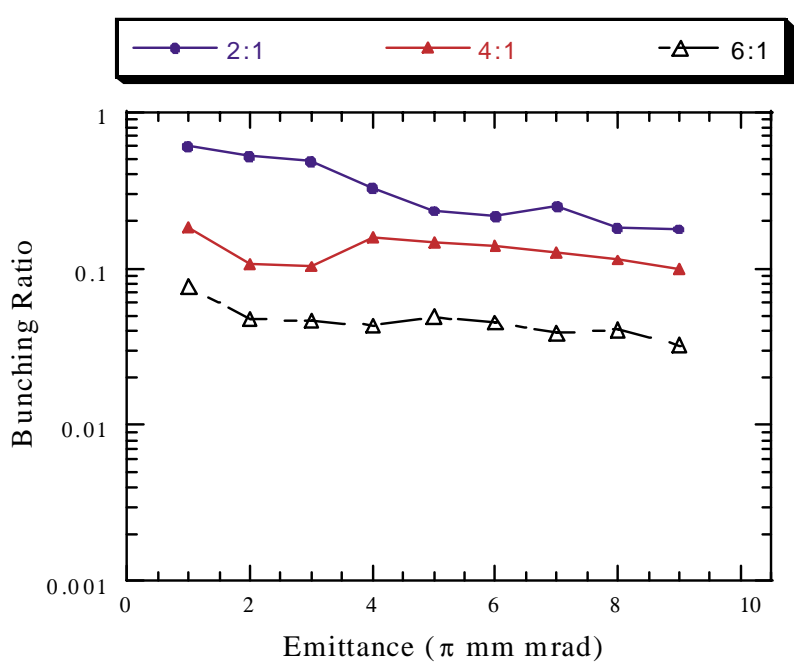

(a)

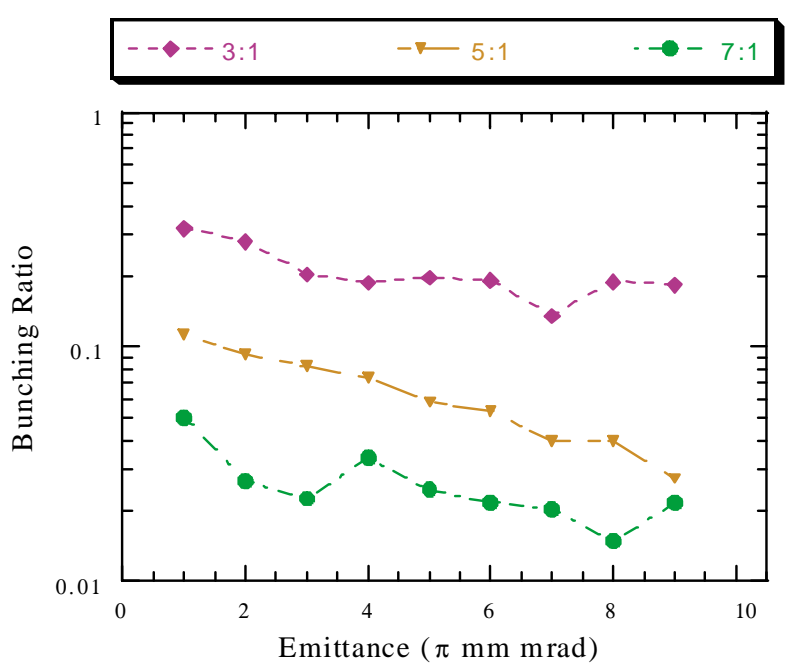

(b)

FIG. 1. (Color) Bunching ratios of the (a) even and (b) odd harmonics to the fundamental versus electron beam emittance as calculated by GINGER.



(a)

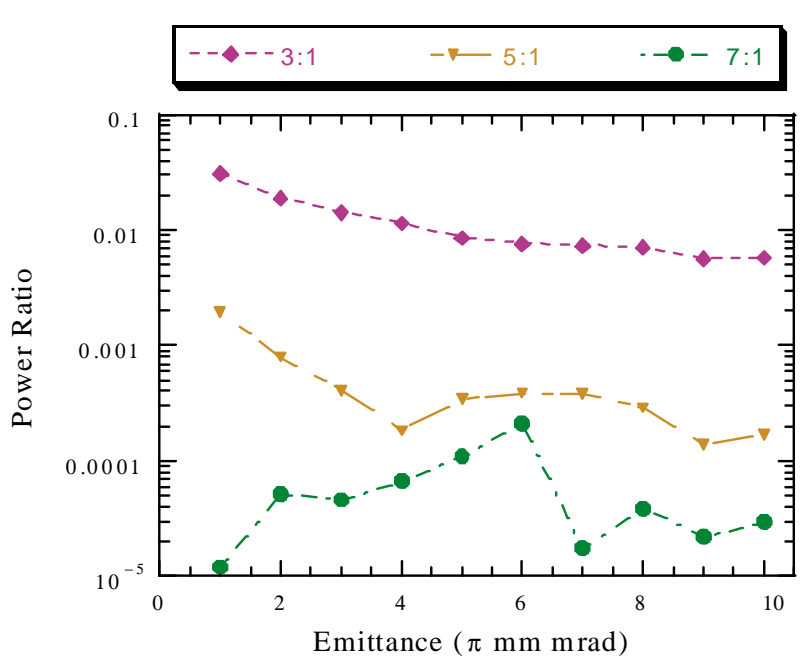

(b)

FIG. 2. (Color) Power ratios of the (a) even and (b) odd harmonics to the fundamental versus electron beam emittance as calculated by MEDUSA. 


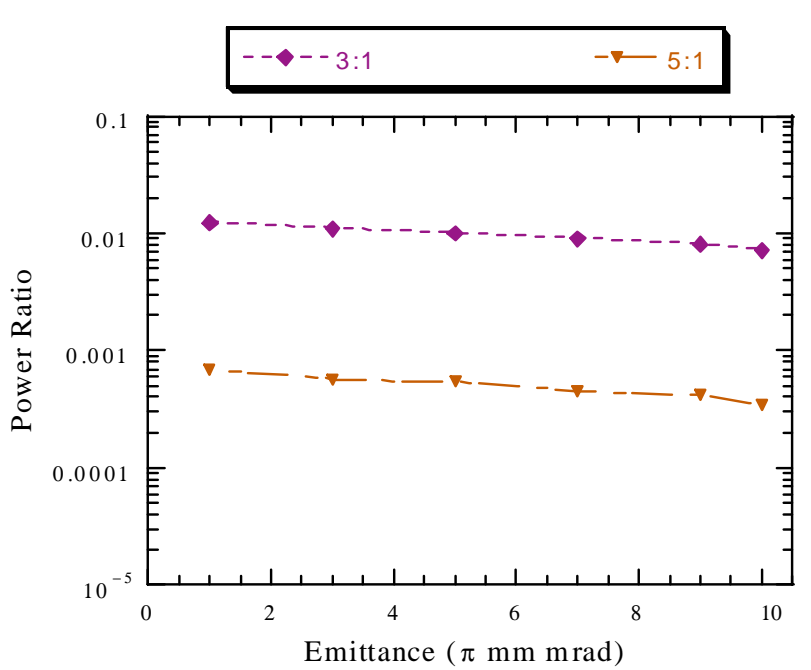

FIG. 3. (Color) Power ratios of the odd harmonics to the fundamental versus electron beam emittance as calculated by PROMETEO.

harmonics do not necessarily correspond to the points at which the harmonic emission is greatest, and this can introduce some fluctuations in the harmonic output as functions of emittance, energy spread, and beam current. In the following graphs, we plot the ratios of the harmonic-tofundamental bunching or output power versus a varying beam parameter, centered around the nominal LEUTL value. Obviously, if these ratios remain more or less constant, the harmonics are not particularly more sensitive than the fundamental to the electron beam quality.

\section{A. Emittance scans}

For the emittance sensitivity scans, the normalized emittance was varied between 1 and $10 \pi \mathrm{mm} \mathrm{mrad,} \mathrm{which}$ leads to the required saturation distance approximately doubling. In Figs. 1(a) and 1(b) GINGER results are shown for the ratio of the bunching fraction at the even and odd harmonics relative to that corresponding to the fundamental wavelength, respectively, up to harmonic number $h=7$. In Figs. 2(a) and 2(b) MEDUSA results are shown for the ratio of output power of the even and odd harmonics relative to that emitted at the fundamental, respectively, up to $h=7$ also. In Fig. 3 the PROMETEO results are shown for the power ratios of the odd harmonics to the fundamental up to $h=5$. Over this limited range, none of these ratios show significant sensitivity to emittance with the exception of the predicted power at the second harmonic as calculated by MEDUSA.

\section{B. Energy spread scans}

For the energy spread scans, the initial electron beam energy spread (Gaussian distribution) was varied between 0.0 and $0.2 \%$, which increased the required saturation distance by $\sim 75 \%$. Figures 4 (a) and 4(b) display the GINGER predictions for the bunching ratios of the even and odd harmonics to the fundamental, respectively, up to $h=7$. Figures 5(a) and 5(b) show the MEDUSA results for the power ratios of the even and odd harmonics to the fundamental, respectively, up to $h=7$. Figure 6 shows the PROMETEO results for the power ratios of the odd harmonics to the fundamental up to $h=5$. The overall results suggest that, for harmonic numbers $h=5$ and smaller, there is not a dramatic loss of bunching or output power for normalized energy spreads over the interval 0.0 to FEL parameter $\rho \sim 0.002$. The GINGER and PROMETEO curves suggest that the higher harmonics tend to be somewhat more sensitive to increased energy spread although the MEDUSA curves show no clear trend in this regard.

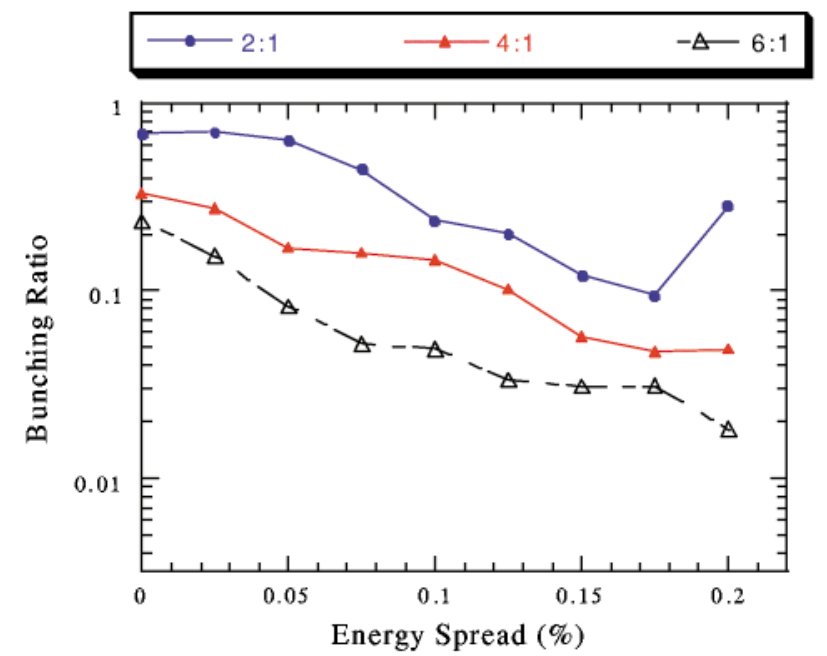

(a)

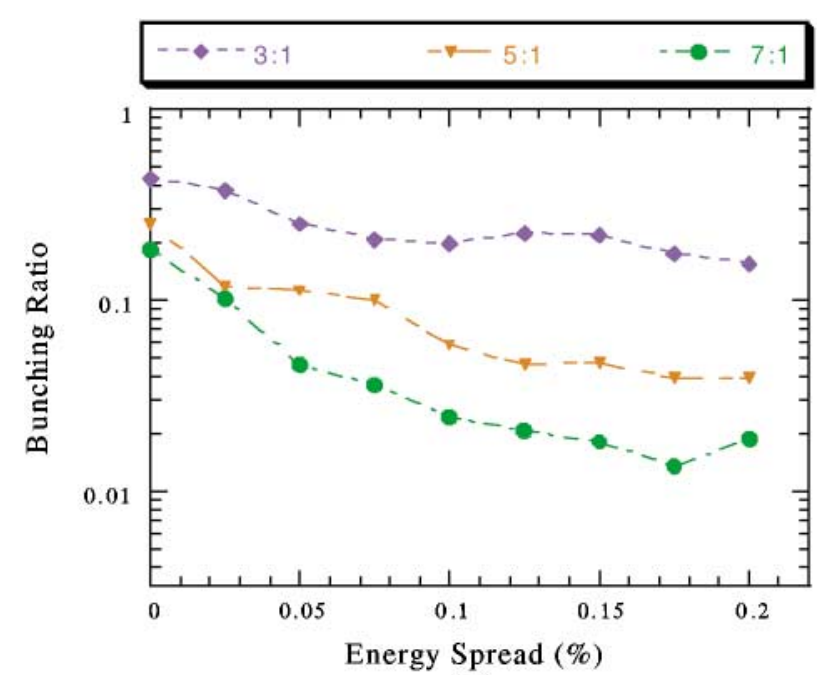

(b)

FIG. 4. (Color) Bunching ratios of the (a) even and (b) odd harmonics to the fundamental using the simulation code GINGER, plotted versus electron beam initial energy spread. 


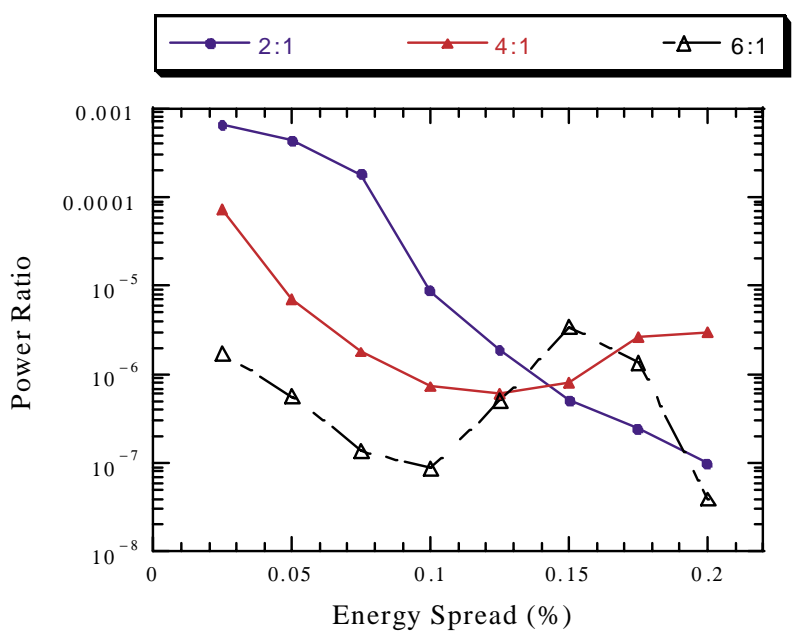

(a)

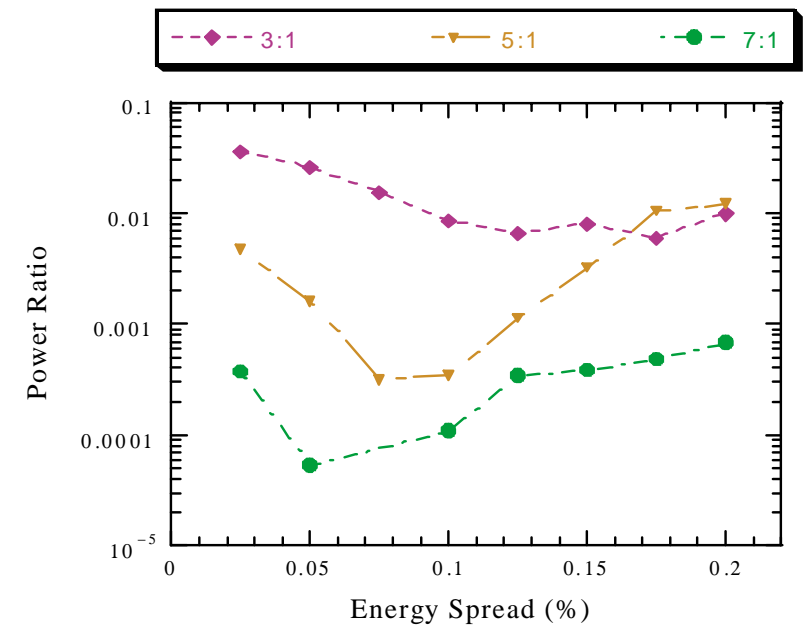

(b)

FIG. 5. (Color) Power ratios of the (a) even and (b) odd harmonics to the fundamental calculated by MEDUSA, plotted versus electron beam initial energy spread.

\section{Electron beam current scans}

For the electron beam current scans, the current was varied between 75 and $300 \mathrm{~A}$. The required distance for saturation is approximately halved over this interval. Figures 7(a) and 7(b) display the GINGER results for the bunching of the even and odd harmonics with the fundamental, respectively, up to $h=7$. Figures 8(a) and 8(b) present the MEDUSA results for the powers of the even and odd harmonics with the fundamental, respectively, up to $h=7$. Here, too, there is no dramatic gain in bunching (all harmonics) or output power (for the odd harmonics), although the MEDUSA-predicted powers for the second and fourth harmonics climb rapidly over the current range of 75 to $200 \mathrm{~A}$. Since the FEL parameter $\rho$ scales only as the one-third power of current, it is not too surprising that there are no



FIG. 6. (Color) Power ratios of the odd harmonics to the fundamental calculated by PROMETEO, plotted versus electron beam initial energy spread. catastrophic falloffs of either harmonic bunching or output power over this limited range.

\section{Bunching results for a SASE-initiated run}

All of the previous results were "amplifier" runs initiated with a weak, totally temporally coherent signal. In order to examine the sensitivity of the predicted bunching to the nature of the input (i.e., true SASE noise versus monochromatic input), we did a relatively long pulse ( $1.5 \mathrm{ps}$ or about twice the slippage time up to first saturation), fully time-dependent GINGER run initiated with shot noise using the nominal LEUTL parameters of Table I. As shown in Fig. 9, the peak time-averaged bunching at the fundamental and harmonics occurs at $z \sim 15 \mathrm{~m}$ with values quite similar to those seen, for example, in Fig. 4 as also expected from the 3D theory [15]. As is typical for SASE runs, the time-dependent bunching is somewhat "spiky" although the relative fluctuation level (when compared to the time-averaged value) lessens as one approaches saturation. Hence, we believe that the sensitivity studies of the previous paragraphs are likely to hold for the SASE-initiated, exponential growth regime.

\section{CONCLUSION}

For LEUTL-like parameters in the exponential gain regime, the predicted bunching and output power for harmonics up to $h=7$ appear to be only moderately more sensitive to electron beam qualities of normalized emittance, energy spread, and peak current than the corresponding quantities at the fundamental wavelength. The underlying reason for this lack of sensitivity in the exponential gain regime is that bunching at higher harmonics is coupled nonlinearly to the growing bunching at the fundamental. Hence, so long as a large fraction of the 


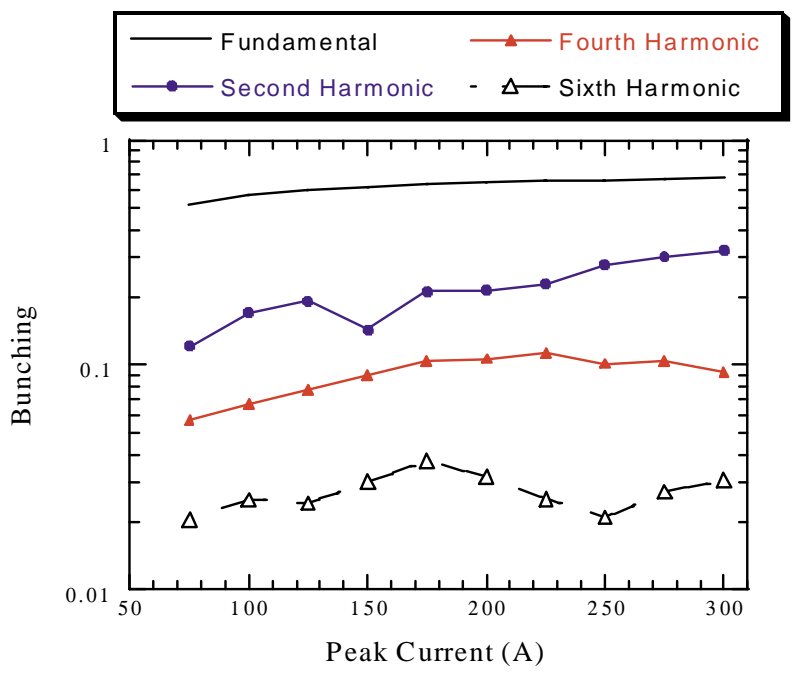

(a)



(b)

FIG. 7. (Color) Bunching fraction as predicted by GINGER for the (a) even and (b) odd harmonics together with that for the fundamental versus electron beam current.

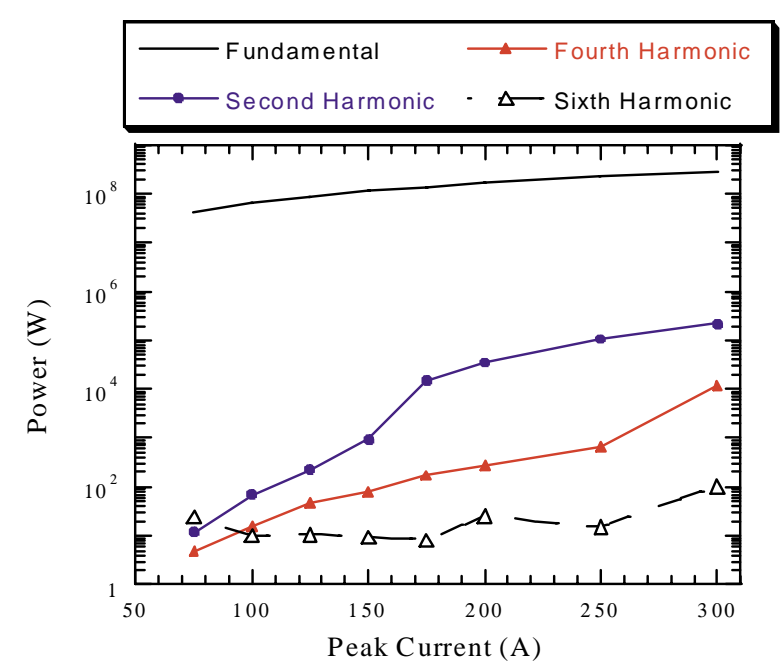

(a)

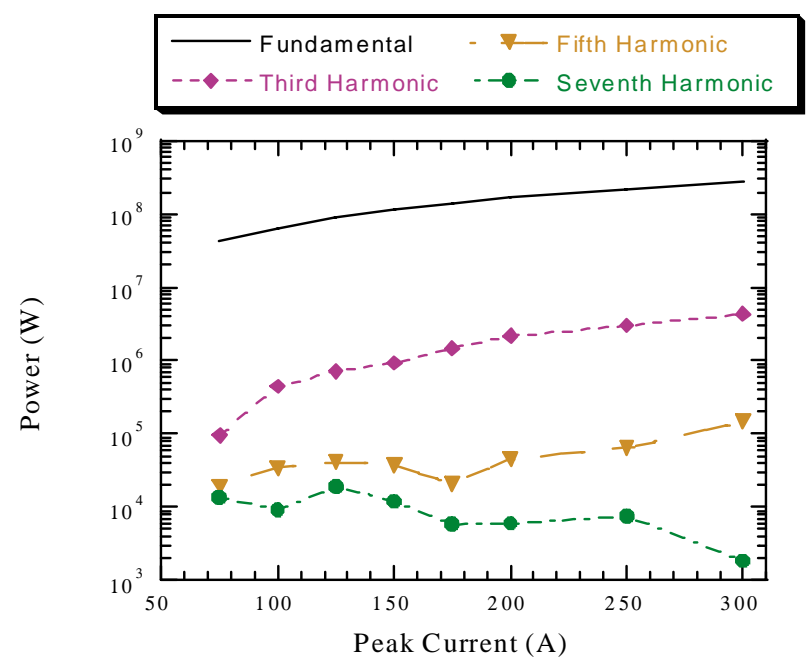

(b)

FIG. 8. (Color) Predicted output powers for the (a) even and (b) odd harmonics together with the fundamental using the simulation code MEDUSA, versus electron beam current.

beam's electrons end up deeply trapped in the FEL's ponderomotive well at the saturation point, there will simultaneously be a large harmonic content to this bunching. If the particles are not deeply trapped, it is likely that gain at the fundamental has been significantly degraded, and the FEL will not operate anywhere close to optimum.

Also note here that the ratios will vary slightly if the analysis is modified by taking the bunching or output power of the harmonics at their own saturation points. This is the next point of investigation for our group, as well as examination of the shorter wavelength regimes.

Experimentally, a number of measurements have been made recently of harmonic output of high-gain harmonic generation and SASE FELs. In the following experiments, the nonlinear harmonic components were dominant. The first, second, and third harmonic intensities were measured in August 2000 at the ANL/BNL collaborative experiment of high-gain harmonic generation at the ATF at BNL [16]. Spectral measurements of the harmonic intensities in a SASE FEL were made at the Institute of Scientific and Industrial Research in Osaka University, Japan $[17,18]$. At the LEUTL SASE FEL, the fundamental and second harmonic intensities, spectra, and coherent transition radiation (CTR) were all measured separately as a function of distance in the exponential gain regime through the undulator line to saturation and beyond. We have also measured the fundamental and second nonlinear harmonic spectra simultaneously [19]. The VISA SASE FEL collaboration 

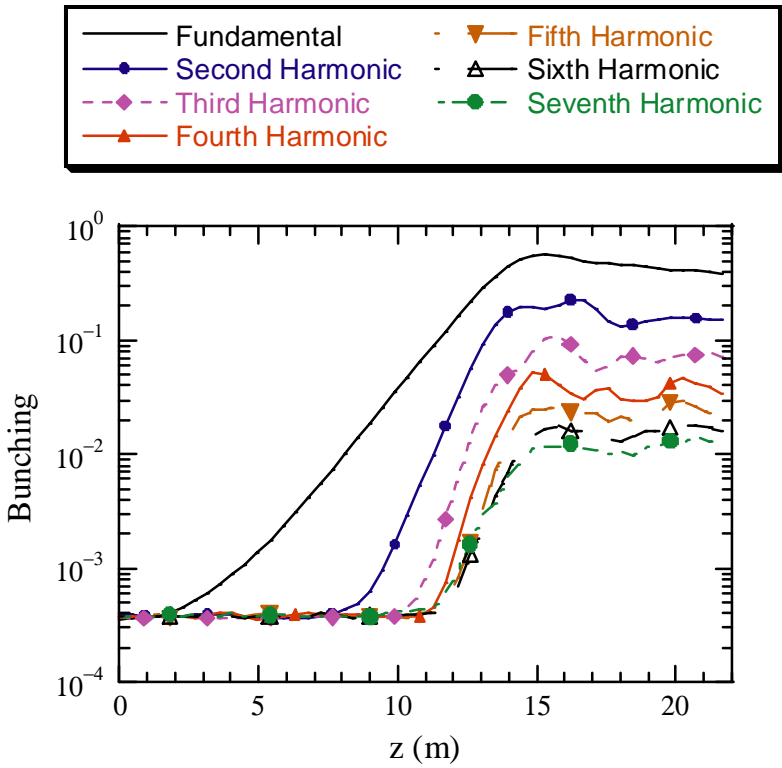

FIG. 9. (Color) GINGER SASE run, bunching as a function of distance for the fundamental and up to the seventh harmonic.

reported measuring the fundamental, second, and third nonlinear harmonic spectra at the point of the fundamental saturation simultaneously. Also, they reported measuring the fundamental, second, and third nonlinear harmonic intensities as a function of distance. Finally, they reported correlating the fundamental and second harmonic CTR with the SASE energy at the point of fundamental saturation (at the end of the undulator) [20].

Nonlinear harmonic generation can be an important aid to achieving shorter wavelengths in next-generation light sources, especially since their growth and eventual saturation vary along with the fundamental bunching. In other words, if the bunching is sufficient at the fundamental, the bunching approaching saturation for the higher harmonics will also be sufficient.

\section{ACKNOWLEDGMENTS}

The work of S. G. Biedron, Z. Huang, K.-J. Kim, and S. V. Milton was supported at Argonne National Laboratory by the U.S. Department of Energy, Office of Basic Energy Sciences under Contract No. W-31-109-ENG-38. The activity and computational work for H.P. Freund was supported by Science Applications International Corporation's Advanced Technology Group under IR\&D subproject No. 01-0060-73-0890-000. The activity of W. M. Fawley was supported at Lawrence Berkeley National Laboratory by the U.S. Department of Energy under Contract No. DE-AC03-76SF00098 and employed computational resources at the National Energy Research Supercomputer Center. The work of H.-D. Nuhn was supported by the U.S. Department of Energy, Office of Basic Energy Sciences, Division of Material Sciences, under Contract No. DE-AC03-76SF00515.
[1] Recently, there has been impressive progress made in the area of high-gain SASE FEL devices operating at wavelengths ranging from the near-infrared to the far ultraviolet. At the Deutsches Elektronen Synchrotron (DESY) in Hamburg, Germany, exponential gain on the Tesla Test Facility FEL has been demonstrated down to wavelengths of $80 \mathrm{~nm}$ [J. Andruszkow et al., Phys. Rev. Lett. 85, 3825 (2000)]. They have also seen saturation [V. Ayvazyan et al., DESY Report No. 01-226; Phys. Rev. Lett. (to be published)]. The Low-Energy Undulator Test Line at the Advanced Photon Source at Argonne National Laboratory has demonstrated both SASE exponential gain and saturation at $530 \mathrm{~nm}$ and $385 \mathrm{~nm}$ [S.V. Milton et al., originally published in Science Express as 10.1126/science.1059955, May 17, 2001; Science 292, 2037-2041 (2001)]. The VISA SASE FEL collaboration achieved SASE saturation at $840 \mathrm{~nm}$ employing the Accelerator Test Facility at Brookhaven National Laboratory [A. Tremaine (private communication)].

[2] A. M. Kondratenko and E. L. Saldin, Sov. Phys. Dokl. 24, 986 (1979).

[3] R. Bonifacio et al., Nucl. Instrum. Methods Phys. Res., Sect. A 293, 627 (1990); G. Dattoli and P. L. Ottaviani, J. Appl. Phys. 86, 5331 (1999); H.P. Freund et al., IEEE J. Quantum Electron. 36, 275 (2000); Z. Huang and K.-J. Kim, Phys. Rev. E 62, 7295 (2000).

[4] L.-H. Yu, Phys. Rev. A 44, 5178 (1991); I. Ben-Zvi et al., Nucl. Instrum. Methods Phys. Res., Sect. A 318, 208 (1992); L.-H. Yu et al., Science 289, 932 (2000).

[5] R. Bonifacio et al., Nucl. Instrum. Methods Phys. Res., Sect. A 296, 787 (1990); F. Ciocci et al., IEEE J. Quantum Electron. 31, 1242 (1995); W. M. Fawley et al., in Proceedings of the IEEE Particle Accelerator Conference, Dallas, TX, 1995 (IEEE, Piscataway, NJ, 1996), p. 219.

[6] W. M. Fawley et al., in Proceedings of the IEEE Particle Accelerator Conference, Dallas, TX, 1995 (Ref. [5]), pp. 219-221; S. G. Biedron, S. V. Milton, and H.P. Freund, Nucl. Instrum. Methods Phys. Res., Sect. A 475, 401 (2001); J. Wu and L.-H. Yu, Nucl. Instrum. Methods Phys. Res., Sect. A 475, 104 (2001).

[7] S. V. Milton et al., in Free Electron Laser Challenges II, edited by Harold E. Bennett and David H. Dowell, SPIE Proceedings Vol. 3614 (SPIE-International Society for Optical Engineering, Bellingham, WA, 1999), pp. 86-95.

[8] See, for example, Linac Coherent Light Source (LCLS) Design Study Report SLAC-R-521, UC-414, Rev. December 1998; Summary of "The workshop on physics of science with the X-Ray FELs," Arcidosso, Italy, 2000, http://www-ssrl.slac.stanford.edu/lcls/Workshops/ 10-15SEP2000/WELCOME.HTML.

[9] H. P. Freund and T. M. Antonsen, Jr., Principles of FreeElectron Lasers (Chapman \& Hall, London, 1986), 2nd ed.

[10] W. M. Fawley, LBNL Report No. LBNL-49625, 2002.

[11] N. M. Kroll, P. L. Morton, and M. R. Rosenbluth, IEEE J. Quantum Electron. 17, 1436 (1981).

[12] H.P. Freund and T.M. Antonsen, Jr., Principles of FreeElectron Lasers (Ref. [9]); H. P. Freund, Phys. Rev. E 52, 5401 (1995); S. G. Biedron, H. P. Freund, and S. V. Milton, in Free Electron Laser Challenges II (Ref. [7]), p. 96-108; H.P. Freund et al., IEEE J. Quantum Electron. 36, 275 (2000). 
[13] G. Dattoli, M. Galli, and P.L. Ottaviani, ENEA Internal Report No. RT/INN/93/09.

[14] D. Prosnitz, A. Szoke, and V. K. Neil, Phys. Rev. A 24, 1436 (1981); see also T. Scharlemann, J. Appl. Phys. 58, 2154 (1985).

[15] Z. Huang and K.-J. Kim, Phys. Rev. E 62, 7295 (2000).

[16] A. Doyuran et al., Phys. Rev. Lett. 86, 5902 (2001).

[17] R. Kato et al., Nucl. Instrum. Methods Phys. Res., Sect. A 475, 334 (2001).

[18] R. Kato et al., "Higher Harmonic Generation Observed in
SASE in the Far-Infrared Region," Nucl. Instrum. Methods Phys. Res., Sect. A (to be published).

[19] S. Biedron et al., "Measurements of Nonlinear Harmonic Generation at the Advanced Photon Source's SASE FEL," Nucl. Instrum. Methods Phys. Res., Sect. A (to be published).

[20] A. Tremaine et al., "Experimental Characterization of SASE FEL Harmonic Radiation at Saturation," Nucl. Instrum. Methods Phys. Res., Sect. A (to be published). 